\title{
Desempenho de Gasolinas em Motor do Ciclo AtKinson
}

\author{
Rogério Nascimento de Carvalho ${ }^{1}$, Antonio Carlos Scardini Villela ${ }^{1}$ e Sérgio William Botero ${ }^{1}$ \\ ${ }^{1}$ Petróleo Brasileiro S.A. \\ E-mails: rogerio.carvalho@petrobras.com.br, antonio.villela@petrobras.com.br, \\ sergio.botero@petrobras.com.br
}

\begin{abstract}
RESUMO
Este artigo apresenta um estudo experimental para avaliação dos efeitos da variação da octanagem e da massa específica de gasolinas em um motor que opera segundo o ciclo Atkinson. Sua principal característica é possuir uma taxa de compressão inferior à taxa de expansão, em função do fechamento tardio da válvula de admissão. Duas diferentes gasolinas foram investigadas e comparadas a uma gasolina comercial brasileira, sendo uma com baixa octanagem e baixa densidade e outra com elevada octanagem e densidade intermediária.
\end{abstract}

Os resultados indicaram que o uso de uma gasolina de baixa octanagem no motor do ciclo Atkinson avaliado, não trouxe riscos significativos de integridade e de funcionamento, uma vez que não houve episódios de detonação, assim como houve comportamento similar de desempenho em comparação à gasolina comum. Apresentou redução das emissões de $\mathrm{CO}_{2} \mathrm{e}$ perda de autonomia no ciclo urbano. Em trabalhos anteriores no Cenpes, observou-se em veículos convencionais do ciclo Otto, que houve perdas de desempenho e/ou eventos importantes de detonação com o uso da gasolina de baixa octanagem. Quanto à gasolina de alta octanagem, não foram observadas diferenças de desempenho, de emissões de $\mathrm{CO}_{2}$ e de eficiência em relação à gasolina comercial.

\section{INTRODUÇÃO}

A indústria da mobilidade global passa por uma importante fase de transformação tecnológica e de costumes, com o principal objetivo de controlar e reduzir as emissões de carbono para a atmosfera. É a chamada era de transição para a matriz de baixo carbono.

Segundo KALGHATGI [1], o transporte atual de pessoas e mercadorias é responsável por aproximadamente $20 \%$ do consumo global de energia primária, por cerca de $23 \%$ das emissões de dióxido de carbono $\left(\mathrm{CO}_{2}\right)$ e por $14 \%$ da emissão global de gases de efeito estufa (GEE), considerando a emissão de metano e outros gases, o que totaliza cerca de 7 bilhões de toneladas de $\mathrm{CO}_{2}$ equivalente.

A frota mundial é composta por aproximadamente 1,2 bilhões de veículos leves de passageiros e 380 milhões de veículos comerciais e esses números tentem a aumentar, principalmente em países como China e Índia. Praticamente 99,9\% desta frota é equipada com motores a combustão interna. Cerca de $95 \%$ da energia utilizada nos transportes é consumida através de 
combustíveis líquidos fósseis e cerca de $60 \%$ de todo petróleo produzido é utilizado na fabricação de combustíveis para motores $[1,2]$.

Várias iniciativas no sentido de reduzir as emissões de $\mathrm{CO}_{2}$, aumentar a eficiência energética dos motores e veículos e mitigar os problemas de poluição local nos grandes centros urbanos vêm se apresentando de forma crescente nos últimos anos. A substituição de combustíveis fósseis por combustíveis renováveis, a eletrificação dos sistemas de propulsão e o incentivo ao uso compartilhado de veículos, são alguns exemplos das mudanças tecnológicas e culturais em andamento. Diversos países já acenam com fortes restrições ao uso dos motores a combustão nas próximas décadas e fomentam a eletrificação de suas frotas.

Entretanto, por questões tecnológicas, econômicas e sociais, projeções indicam que a fase de transição para a eletrificação veicular deverá perdurar ainda por algumas décadas e ocorrer em velocidades distintas nas diferentes regiões do mundo. Por isso, as próximas gerações dos motores a combustão interna deverão incorporar elevados níveis de evolução tecnológica para serem capazes de atingir os objetivos estipulados pelas novas regulamentações.

JOHNSON e JOSHI [3,4] apresentam uma revisão abrangente das tecnologias atualmente em desenvolvimento para a redução das emissões de $\mathrm{CO}_{2}$, tanto para os motores de aplicação nos veículos leves, quanto para os pesados. Especificamente para os veículos leves a gasolina, a Tabela 1 lista algumas das mais promissoras tecnologias na visão destes autores, incluindo as estimativas de redução de $\mathrm{CO}_{2}$ em comparação a um motor de referência, com injeção direta de gasolina (GDI), estequiométrico e turboalimentado, bem como o nível de maturidade tecnológica de cada desenvolvimento.

Tabela 1. Tecnologias para redução das emissões de $\mathrm{CO}_{2}$ de veículos leves (adaptado de [4]).

\begin{tabular}{|l|c|c|}
\hline \multicolumn{1}{|c|}{ Tecnologia de Motor } & $\begin{array}{c}\text { Redução } \\
\text { de } \mathbf{C O}_{2}\end{array}$ & Nível de Maturidade \\
\hline GDI Estequiométrico, Turboalimentado & referência & Implantado \\
\hline $\begin{array}{l}\text { Ciclo Atkinson com Comando de Válvulas Variável } \\
\text { (VVT) }\end{array}$ & $3-5 \%$ & Implantado \\
\hline Start-Stop Avançado & $2-5 \%$ & Implantado \\
\hline Desativação Dinâmica de Cilindros & $2-10 \%$ & Implantado \\
\hline GDI Estratificado & $10-20 \%$ & Implantado \\
\hline Taxa de Compressão Variável & $10 \%$ & Implantado \\
\hline $\begin{array}{l}\text { Ignição por Compressão Controlada por Centelha } \\
\text { (SpCCI) }\end{array}$ & $20-30 \%$ & Implantado \\
\hline Injeção de Água & $5-10 \%$ & Em desenvolvimento \\
\hline $\begin{array}{l}\text { Recirculação de Gases da Exaustão (EGR) } \\
\text { Dedicado }\end{array}$ & $15-20 \%$ & Engenharia avançada \\
\hline $\begin{array}{l}\text { Ignição por Compressão Controlada por Reatividade } \\
\text { (RCCI) }\end{array}$ & $20-30 \%$ & Engenharia avançada \\
\hline Pistões Opostos, Dois Tempos & $25-35 \%$ & Engenharia avançada \\
\hline $\begin{array}{l}\text { Ignição por Compressão com Injeção Direta de } \\
\text { Gasolina (GDCI) }\end{array}$ & $15-25 \%$ & Engenharia avançada \\
\hline
\end{tabular}

Algumas destas tecnologias vêm recentemente sendo anunciadas como estando na iminência de serem disponibilizadas comercialmente, como o motor SkyActive-X da Mazda, com o princípio de ignição por compressão controlada por centelha (Spark Contolled Compression 
Ignition - SpCCI), que utiliza ao mesmo tempo conceitos de combustão dos motores de ignição por centelha e por compressão. A empresa anuncia ganhos potenciais de eficiência entre 20 e $30 \%[5]$.

Outro desenvolvimento recentemente anunciado é do motor por pistões opostos, da empresa Achates Power. Esta tecnologia é de um motor dois tempos no qual dois pistões se movimentam em sentidos opostos dentro de um mesmo cilindro, comprimindo e expandindo uma câmara de combustão formada por seus topos. Este motor funciona por compressão de diesel e estima-se um ganho de eficiência da ordem de 25 a $35 \%$ [6].

A tecnologia de taxa de compressão variável foi anunciada recentemente pela fabricante Infiniti como disponível comercialmente em 2019. Esta tecnologia permite que a taxa de compressão do motor seja modificada em função do regime de operação do motor ou do tipo de combustível utilizado, otimizando a combustão para cada condição [7].

O uso de ciclos não convencionais em substituição ao ciclo Otto, em determinadas condições de operação, é outra solução que vem sendo empregada aos motores para o aumento da eficiência energética. Os mais comumente aplicados são os ciclos Atkinson e Miller que, com o uso de sistemas de comando de válvulas variável (VVT), atrasam o fechamento da válvula de admissão, fazendo com que a razão de compressão seja menor do que a razão de expansão, o que favorece a eficiência térmica de conversão da energia do combustível em trabalho no motor. No entanto, uma vez que a quantidade de energia disponível no volume de mistura arcombustível que efetivamente participa da combustão é menor, menos trabalho útil é gerado, resultando em níveis mais baixos de desempenho em termos de potência e torque do motor.

\section{O CICLO ATKINSON}

Em 1882, o engenheiro inglês James Atkinson idealizou um motor a combustão interna cuja principal característica era possuir o curso de expansão maior do que o de compressão. Seu objetivo era alcançar melhores eficiências em relação ao motor apresentado alguns anos antes por Nikolaus Otto. Entretanto, o projeto original deste motor (Fig.1), a despeito da maior eficiência, apresentava alta complexidade de construção e de funcionamento e assim, não se difundiu como aconteceu com o projeto de Otto [8].

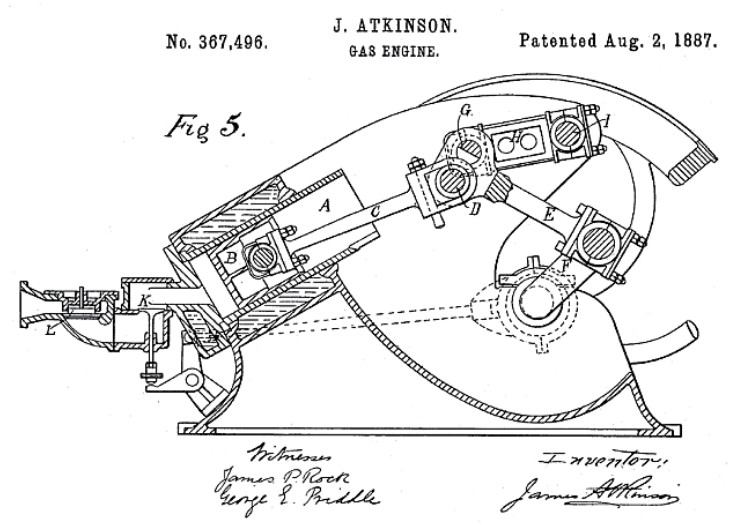

Fig. 1. Projeto original do motor patenteado por James Atkinson [9].

As figuras 2 e 3 apresentam conceitualmente os tempos de funcionamento e os diagramas pressão $x$ volume que representam, respectivamente, os ciclos Otto e Atkinson. 

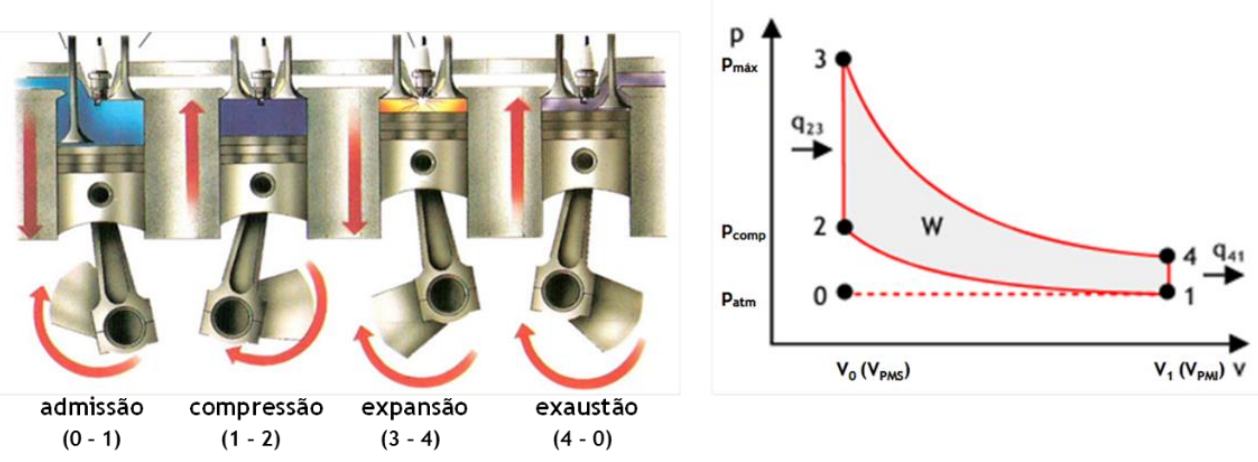

Fig. 2. Tempos de funcionamento do motor do ciclo Otto (adaptado de [10]).

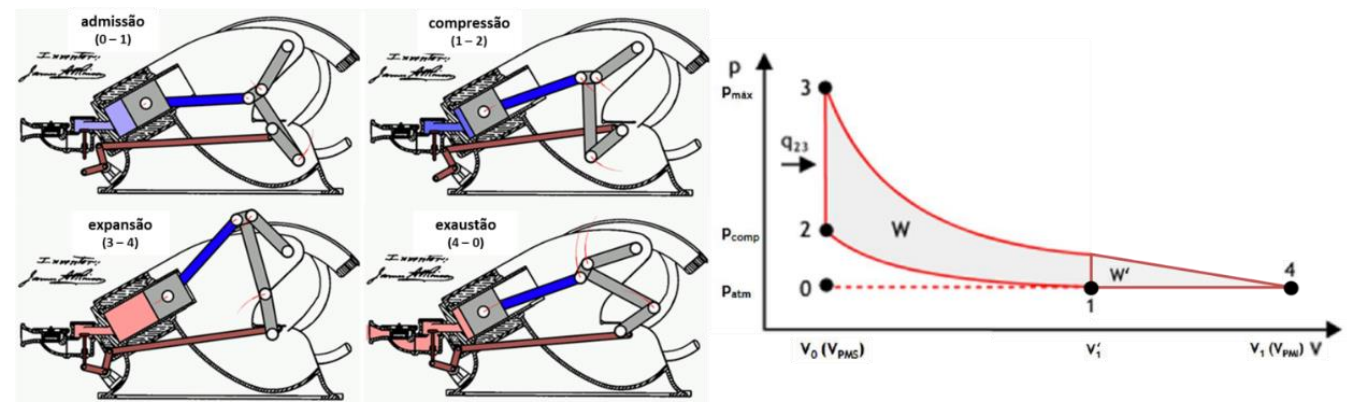

Fig. 3. Tempos de funcionamento do motor do ciclo Atkinson (adaptado de [10,11]).

Assumindo-se idealmente que os motores apresentados nas figuras 2 e 3 possuam entre si os mesmos cursos de compressão (1-2) e as mesmas quantidades de energia $\left(\mathrm{q}_{23}\right)$ geradas na combustão, percebe-se dos diagramas $p$ - $V$ que a parcela $\left(\mathrm{q}_{41}\right)$ de calor rejeitada pela exaustão no ciclo Otto pode ser convertida em trabalho útil adicional (W') com o prolongamento do curso de expansão (3-4) característico do ciclo Atkinson. Obtém-se assim uma maior eficiência térmica de conversão de energia do combustível em trabalho.

Modernamente, os conceitos propostos por Atkinson vêm sendo aplicados em motores originalmente projetados para o funcionamento segundo do ciclo Otto, com o objetivo de alcançar melhores condições de eficiência. Com o uso de sistemas de comando de válvulas variável e gerenciamentos eletrônicos adequados, uma estratégia para simular o ciclo Atkinson é realizar mais tardiamente o fechamento da válvula de admissão, durante o deslocamento do pistão no tempo da compressão.

Ou seja, na condição de ciclo Atkinson, o início efetivo da compressão se dá em uma posição intermediária do curso do pistão, no momento em que a válvula de admissão é fechada, fazendo com que uma parte do curso ocorra sem a compressão efetiva. Com isso, reduzem-se as perdas por bombeamento, o que também favorece uma melhor eficiência mecânica do motor. Após a compressão, o tempo da expansão ocorre em todo o curso do pistão, o que resulta em um melhor aproveitamento da energia da combustão que é transformada em trabalho, gerando menores níveis de calor rejeitado pelo escapamento e, por consequência, melhor eficiência térmica de conversão.

Em contrapartida, uma vez que o volume de mistura ar-combustível que efetivamente participa da combustão é menor do que durante o funcionamento na condição de ciclo Otto, a quantidade 
de energia disponível para gerar trabalho também é menor, resultando em níveis mais baixos de desempenho em termos de potência do motor.

Diversos fabricantes têm aplicado em seus veículos, motores capazes de operar alternativamente no ciclo Otto e no ciclo Atkinson, dependendo da condição momentânea de rotação e carga. Assim, quando não são requeridos níveis elevados de torque e potência, o motor funciona com o fechamento tardio das válvulas de admissão, aproveitando a característica de maiores eficiências em rotações mais baixas do ciclo Atkinson. Quando o regime de funcionamento do motor exige cargas e rotações mais elevadas, o motor passa a operar segundo o ciclo Otto, obtendo melhores desempenhos em detrimento da eficiência.

Outra aplicação na qual os motores do ciclo Atkinson estão se difundindo é na unidade motora a combustão dos veículos híbridos. Nestes veículos, o motor a combustão opera normalmente na configuração de ciclo Atkinson, uma vez que é usado como complemento ao motor elétrico quando se requer um nível mais elevado de potência do conjunto, ou para recarga da bateria. Em ambos os casos, o motor a combustão opera na condição de melhor eficiência, não sendo requeridos níveis elevados de desempenho, o que viabiliza o uso do ciclo Atkinson.

\section{METODOLOGIA EXPERIMENTAL}

O objetivo deste trabalho foi investigar experimentalmente a sensibilidade de um veículo híbrido, equipado com motor do ciclo Atkinson, às variações de propriedades da gasolina brasileira, principalmente em relação à octanagem e à densidade. Assim, três diferentes gasolinas foram aplicadas e avaliadas em relação ao desempenho em retomadas de velocidade, potência e consumo específico do motor a combustão em regime permanente, autonomia urbana e emissões de $\mathrm{CO}_{2}$, além de ocorrências de detonação. A Tabela 2 apresenta algumas das principais propriedades das gasolinas de teste.

Tabela 2. Principais propriedades físico-químicas das gasolinas de teste.

\begin{tabular}{|l|c|c|c|c|}
\hline \multicolumn{1}{|c|}{ Propriedade } & Método & BAIXA_OCT & MÉDIA_OCT & ALTA_OCT \\
\hline $\begin{array}{l}\text { Teor de Etanol Anidro } \\
(\% \mathrm{v} / \mathrm{v})\end{array}$ & NBR 13992 & 26 & 26 & 25 \\
\hline $\begin{array}{l}\text { Massa Específica a } \\
20^{\circ} \mathrm{C}\left(\mathrm{kg} / \mathrm{m}^{3}\right)\end{array}$ & ASTM D4052 & 713,4 & 748,8 & 734,8 \\
\hline PCI $(\mathrm{MJ} / \mathrm{kg})$ & ASTM D4809 & 39,1 & 38,7 & 38,8 \\
\hline$\% \mathrm{C}(\% \mathrm{~m} / \mathrm{m})$ & ASTM D5291 & 73,6 & 75,4 & 76,0 \\
\cline { 1 - 3 }$\% \mathrm{H}(\% \mathrm{~m} / \mathrm{m})$ & ASTM D5622 & 14,7 & 13,4 & 13,7 \\
\cline { 4 - 6 }$\% \mathrm{O}(\% \mathrm{~m} / \mathrm{m})$ & ---- & 12,9 & 11,2 & 10,3 \\
\hline Relação A/C esteq. & ASTM D2700 & 84,0 & 84,0 & 12,9 \\
\hline MON & ASTM D2699 & 90,2 & 97,1 & 105,0 \\
\hline RON & ---- & 87,1 & 90,6 & 98,3 \\
\hline IAD & & & & \\
\hline
\end{tabular}

Pode-se observar que foram aplicadas: uma formulação com baixa octanagem RON 90 e MON 84 (IAD 87) e baixa massa específica de $713,4 \mathrm{~kg} / \mathrm{m}^{3}$, identificada como "BAIXA_OCT"; uma gasolina comercial, com octanagem RON 97 e MON 84 (IAD 90,6) e massa específica de 748,8 $\mathrm{kg} / \mathrm{m}^{3}$, identificada por "MÉDIA_OCT"; uma gasolina premium com elevada octanagem RON 105 e MON 91,6 (IAD 98,3) e massa específica de $734,8 \mathrm{~kg} / \mathrm{m}^{3}$, identificada por "ALTA_OCT". 


\subsection{Desempenho em Retomadas de Velocidade}

Os ensaios de retomadas de velocidade têm por objetivo avaliar o desempenho de um veículo em condições de aceleração transiente a plena carga em diferentes regimes de funcionamento do motor. O intervalo de tempo decorrido entre o início da aceleração em uma determinada velocidade e o momento em que o veículo atinge a velocidade final estabelecida é o parâmetro avaliado. Quanto menor for este intervalo de tempo, melhor será o desempenho do veículo neste ensaio.

Os ensaios de retomada de velocidade foram realizados em dinamômetro de chassi seguindo metodologia experimental desenvolvida internamente no Cenpes [12] e os tempos foram medidos em dois intervalos: entre 40 e $80 \mathrm{~km} / \mathrm{h}$ e entre 60 e $100 \mathrm{~km} / \mathrm{h}$. Neste procedimento, as medições em laboratório apresentam menores variações das condições ambientais em comparação aos ensaios executados em pista de teste.

Para eliminar a influência do tempo de reação do motorista e obter uma maior precisão nos resultados, este procedimento consiste na medição dos tempos de retomada de velocidade em plena carga. Para tanto, o dinamômetro de chassi é configurado na velocidade inicial do intervalo de retomada, no modo de velocidade constante. A seguir, o motorista aciona o pedal em curso máximo com o dinamômetro ainda mantendo a velocidade configurada. Após a estabilização de velocidade e plena carga é acionado o modo de simulação do dinamômetro com a sua respectiva curva de coast down e o veículo inicia a aceleração até que atinja a máxima velocidade do intervalo de retomada.

Para que seja possível uma comparação direta entre os tempos obtidos com diferentes combustíveis, os resultados devem ser colocados em uma mesma base de temperatura e pressão. Para tanto foi utilizada uma metodologia, adaptada da norma brasileira ABNT NBR 1585 [13] para ensaios de potência em motores, na qual se propõe que a variação do tempo de retomada é inversamente proporcional à variação da potência do motor. Esta hipótese foi formulada com base no comportamento linear da velocidade em ensaios experimentais de retomada de velocidade, com aceleração aproximadamente constante [12].

\subsection{Desempenho em Regime Permanente}

Os ensaios de desempenho em regime permanente têm por objetivo avaliar diferentes parâmetros de funcionamento do motor a combustão interna e assim avaliar o efeito de diferentes combustíveis nestes atributos. Nestes ensaios foram avaliados a potência do motor a combustão e o consumo específico de combustível, além de análises de detonação.

Neste procedimento desenvolvido internamente no Cenpes, o veículo foi instalado no dinamômetro de chassi e o acelerador acionado totalmente, colocando o motor no regime de plena carga. $\mathrm{O}$ dinamômetro foi configurado para manter a velocidade fixa na condição estabelecida de teste, mantendo o veículo em regime permanente nesta velocidade. Após a estabilização inicial, foram realizadas as aquisições dos parâmetros monitorados por um período de 15 segundos. 
Os ensaios foram realizados nas velocidades de 40, 60, 80 e $100 \mathrm{~km} / \mathrm{h}$, que coincidem com as velocidades iniciais e finais dos ensaios de retomada de velocidade e cobrem uma faixa de rotação entre 3.500 e $4.500 \mathrm{rpm}$. Para cada velocidade foram realizadas pelo menos cinco repetições do procedimento de 15 segundos, tendo como parâmetro para o ponto de partida a estabilização da temperatura do ar de admissão.

Para a obtenção dos parâmetros medidos, foram utilizados diferentes sistemas de aquisição. Um sistema desenvolvido internamente em LabView, interligado a um equipamento de medição de tensão e corrente da bateria do veículo híbrido, possibilitou a medição da potência líquida consumida pelo motor elétrico durante o ensaio. Neste mesmo sistema de aquisição, foram obtidas as informações de potência do dinamômetro de chassi.

Informações da central eletrônica do veículo como avanço de ignição, temperatura do ar de admissão, vazão mássica de ar e fator lambda foram obtidos a partir de um sistema de leitura do OBD. As condições de pressão atmosférica e umidade relativa na sala de testes foram obtidas do sistema de monitoramento do ambiente e o consumo instantâneo de combustível foi anotado a partir do painel do veículo ao final de cada aceleração. Adicionalmente, os sinais do sensor de detonação do motor foram adquiridos a cada aceleração para posterior tratamento.

A seguir, são detalhados os procedimentos de avaliação de cada um dos parâmetros de funcionamento do motor.

\section{- POTÊNCIA DO MCI}

A potência gerada por cada combustível no motor a combustão interna foi calculada de forma indireta a partir das medições da potência global, obtida no dinamômetro de chassi, e da potência disponibilizada pelo motor elétrico. Ambas foram adquiridas pelo sistema em LabView configurado especificamente para este fim. A potência do motor elétrico foi obtida por um sistema de medição de corrente e tensão de veículos elétricos instalado no veículo conforme ilustram as figuras 4(a) e 4(b).

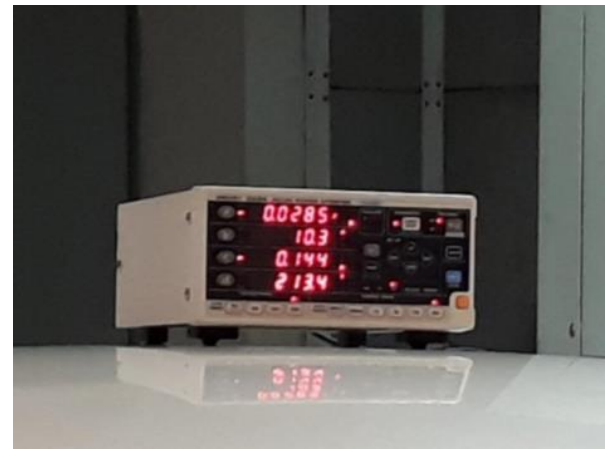

(a)

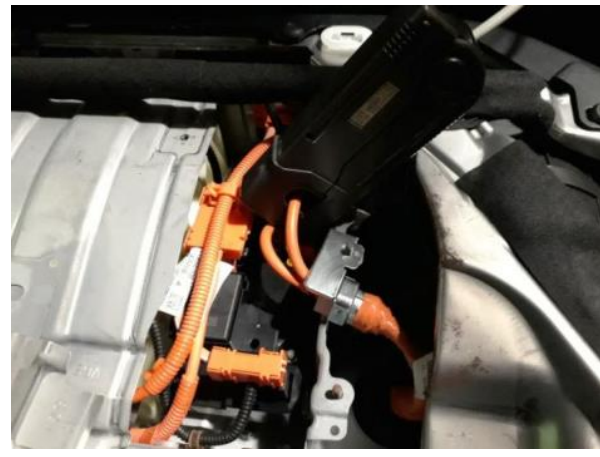

(b)

Fig. 4. (a) Sistema de medição para veículos elétricos; (b) Sensor de corrente e tensão instalado no sistema elétrico do veículo híbrido.

Neste trabalho, assumiu-se que a potência global do veículo medida no dinamômetro de chassi, seria o somatório das potências geradas pelo motor a combustão interna e pelo motor elétrico. Não foram consideradas neste cálculo as perdas internas do 
veículo e do dinamômetro, que foram assumidas constantes e repetitivas em todos os ensaios.

Desta forma, a potência do motor a combustão interna em cada ensaio foi calculada pela diferença entre os valores medidos de potência no dinamômetro e no motor elétrico. $\mathrm{O}$ valor resultante foi corrigido para as condições de umidade relativa e pressão atmosférica seguindo o procedimento da norma ABNT NBR 1585 [13] para ensaios de potência em motores.

\section{- CONSUMO ESPECÍFICO DE COMBUSTÍVEL}

O consumo específico de combustível, em $\mathrm{g} / \mathrm{kW} . \mathrm{h}$, pode ser obtido pela razão entre a vazão mássica de combustível e a potência do motor a combustão interna, obtida conforme procedimento anteriormente descrito.

A vazão mássica de combustível foi obtida a partir da razão entre a vazão mássica de ar admitido e o produto do fator lambda $x$ razão ar/combustível estequiométrica do combustível. Para padronização das condições ambientes dos ensaios, a vazão mássica de ar foi corrigida a partir da metodologia de Antoine [14], que leva em conta os valores de umidade ambiente e temperatura do ar de admissão.

\section{- DETONAÇÃO}

A avaliação de ocorrências de detonação durante a realização dos ensaios de desempenho a plena carga em regime permanente foi realizada a partir do registro do sinal do sensor de detonação. Foram registrados os arquivos durante cada aceleração para todas as condições de velocidades com os três combustíveis.

Este procedimento foi desenvolvido internamente no Cenpes. O processamento dos sinais de ruído foi realizado em um software comercial específico para este fim, para evidenciar o ruído característico da detonação de forma mais clara. Este processamento foi realizado conforme a seguintes etapas:

- Amplificação das frequências entre 5 e $10 \mathrm{kHz}$, típicas do ruído provocado pelo fenômeno da detonação [15];

- Aplicação de filtros passa-baixa e passa-alta, de forma que as frequências típicas fossem preservadas;

- Minimização do ruído de fundo através de uma função específica do software;

- Identificação de picos com intensidade diferenciada em relação ao padrão de sinal obtido no arquivo processado, também através de função específica do software. Estes picos evidenciam a ocorrência de detonação.

As figuras 5 e 6 apresentam o comportamento típico dos sinais após este processamento, respectivamente para um motor com e sem a ocorrência de detonação. A intensidade da detonação foi classificada de acordo com a magnitude da escala adimensional apresentada pelo software de tratamento do sinal. 
Esta escala foi estabelecida com base em procedimento de detonação audível e buscou correlacionar a magnitude do pico com a intensidade audível da detonação ocorrida nos veículos.

- Até 0,5 - Traços;

- Entre 0,5 e 1,0 - Leve;

- Entre 1,0 e 2,0 - Média;

- Maior do que 2,0 - Severa.

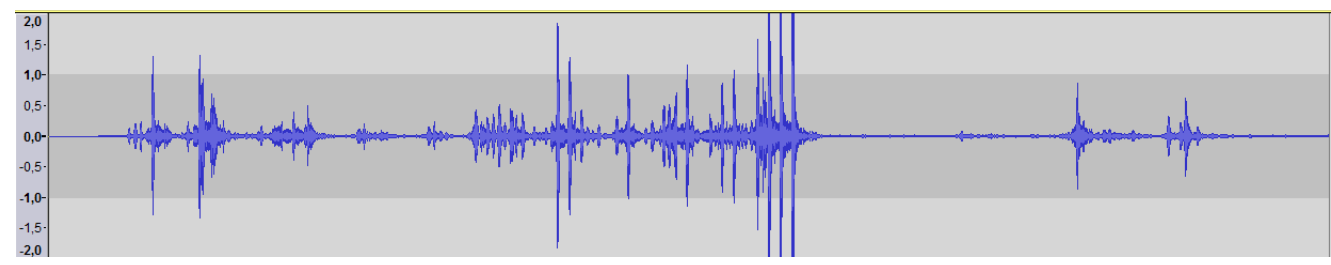

Fig. 5. Comportamento típico do sinal de ruído do veículo com a ocorrência de detonação.

Com esta metodologia é possível identificar visualmente todas as possíveis ocorrências de detonação, representadas graficamente pelos picos de maiores intensidade, sendo possível ainda avaliar a severidade da detonação ocorrida.

Na figura 5 é possível facilmente identificar vários eventos de detonação nos mais variados níveis, conforme a escala acima. Na figura 6 é apresentado um padrão típico de funcionamento do veículo sem ocorrências de detonação, no qual não de identificam os picos característicos destes eventos.

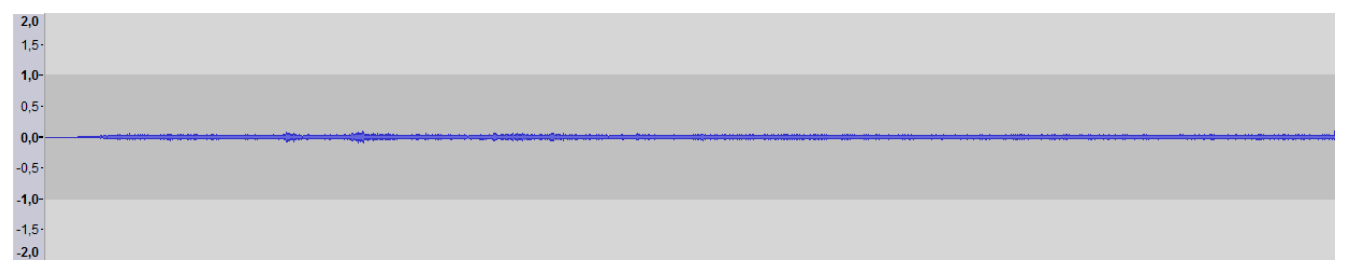

Fig. 6. Comportamento típico do sinal de ruído do veículo sem a ocorrência de detonação.

\subsection{Emissões de $\mathrm{CO}_{2}$ e Autonomia Urbana}

As medições das emissões de $\mathrm{CO}_{2}$ e de autonomia urbana foram executadas seguindo a norma ABNT NBR 16567 [16] que rege o procedimento de testes para veículos híbridos. No cálculo da autonomia urbana, a fórmula do balanço de carbono foi aplicada na sua forma geral (Eq. 1), na qual são levados em consideração os valores de fração de carbono $\left(\% \mathrm{C}_{\mathrm{GAS}}\right.$ e $\left.\% \mathrm{C}_{\mathrm{THC}}\right)$ e de densidade das gasolinas $\left(\rho_{\mathrm{GAS}}\right)$, uma vez que as formulações testadas apresentam variações significativas destes parâmetros entre si, conforme apresentado na Tabela 2.

$$
A u t=\frac{\left(\% C_{G A S} \cdot \rho_{G A S} \cdot \% V_{G A S}^{\prime}\right)+\left(4,1102 \cdot \% V_{E T O H}^{\prime}\right)}{\left(\% C_{T H C} \cdot m_{T H C}\right)+\left(0,4288 \cdot m_{C O}\right)+\left(0,2729 \cdot m_{C O_{2}}\right)}
$$




\section{RESULTADOS}

Em todas as avaliações realizadas neste estudo, a gasolina comercial "MÉDIA_OCT" foi adotada como referência e as gasolinas "BAIXA_OCT" e "ALTA_OCT" foram comparadas a ela. As comparações entre as médias das repetições foram realizadas a partir da teste estatístico $t$ de Student ao nível de confiança de $95 \%$ (p-level < 0,05). Os resultados são apresentados de forma adimensional em gráficos do tipo Box-Plot, sendo 1,00 o valor atribuído à média dos resultados obtidos com a gasolina “MÉDIA_OCT”. Somente são apresentados numericamente os resultados nos casos em que foram observadas diferenças estatisticamente significativas.

\subsection{Desempenho em Retomadas de Velocidade}

As figuras 7 e 8 apresentam graficamente os resultados médios obtidos para os tempos de retomadas de velocidade nos intervalos estabelecidos e suas respectivas variações. Foram realizados pelo menos dez ensaios válidos com cada combustível nos dois intervalos de velocidade.

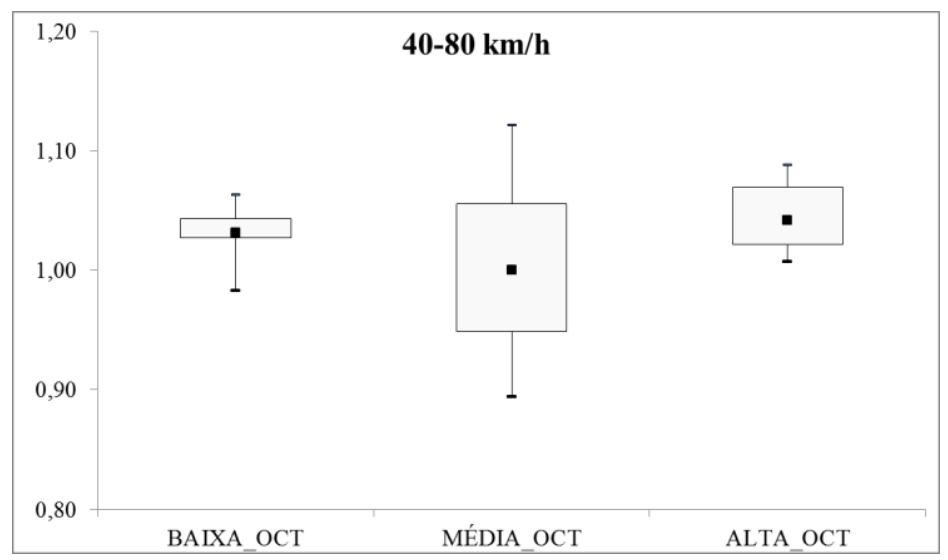

Fig. 7. Resultados dos ensaios de retomadas de velocidade de 40 a $80 \mathrm{~km} / \mathrm{h}$.

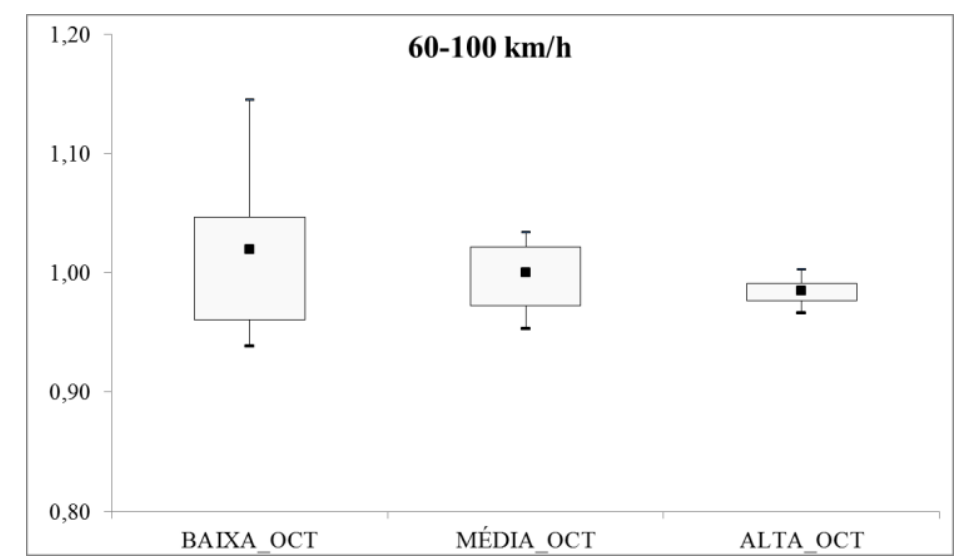

Fig. 8. Resultados dos ensaios de retomadas de velocidade de 60 a $100 \mathrm{~km} / \mathrm{h}$.

Estes resultados indicam que não houve diferenças estatisticamente significativas entre as médias dos ensaios, o que significa que não houve perdas de desempenho do veículo de testes com o uso da gasolina de baixa octanagem em comparação à gasolina comum, nem ganhos com a gasolina de alta octanagem. 


\subsection{Potência do MCI em Regime Permanente}

As figuras 9 a 12 apresentam graficamente os resultados médios da potência do MCI e seus respectivos desvios. Nota-se nestes resultados que nas velocidades de 40, 80 e $100 \mathrm{~km} / \mathrm{h}$ não foram encontradas diferenças estatisticamente significativas.

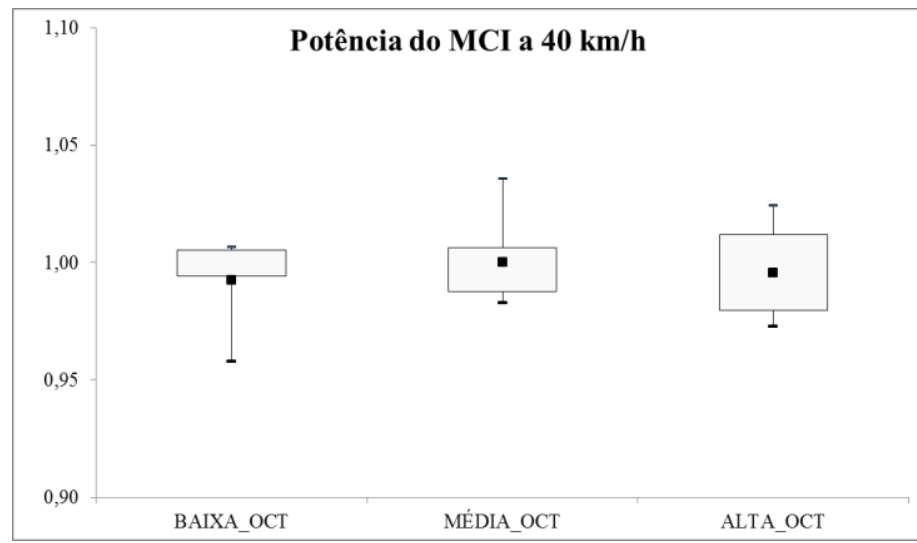

Fig. 9. Resultados dos ensaios de potência do MCI a $40 \mathrm{~km} / \mathrm{h}$.

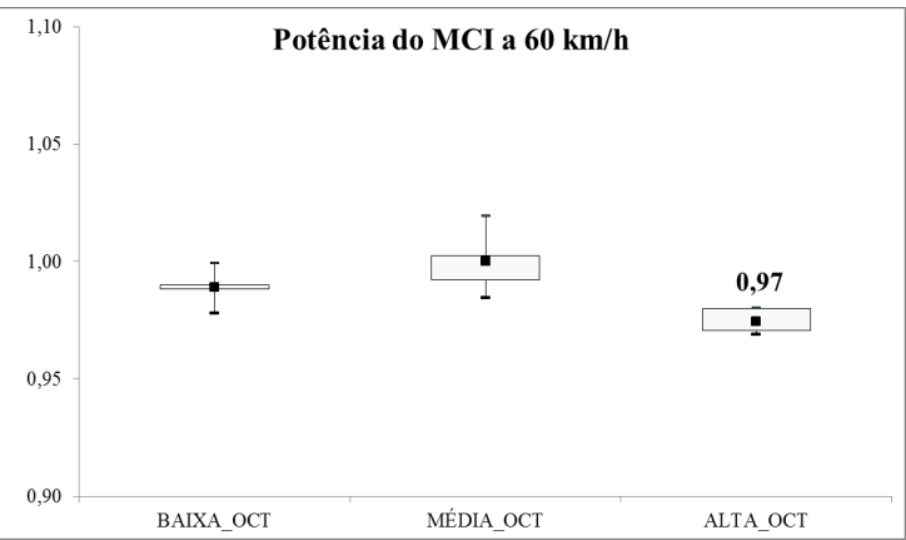

Fig. 10. Resultados dos ensaios de potência do MCI a $60 \mathrm{~km} / \mathrm{h}$.

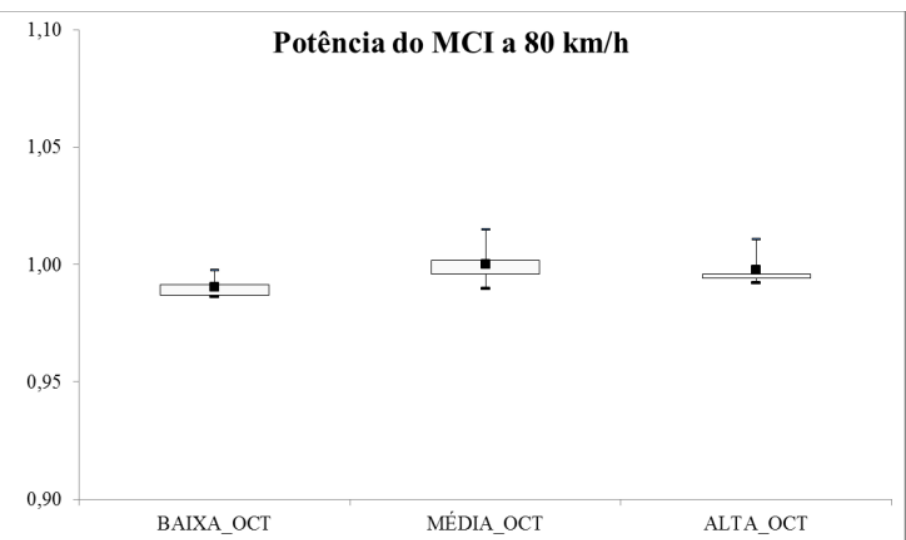

Fig. 11. Resultados dos ensaios de potência do MCI a $80 \mathrm{~km} / \mathrm{h}$. 


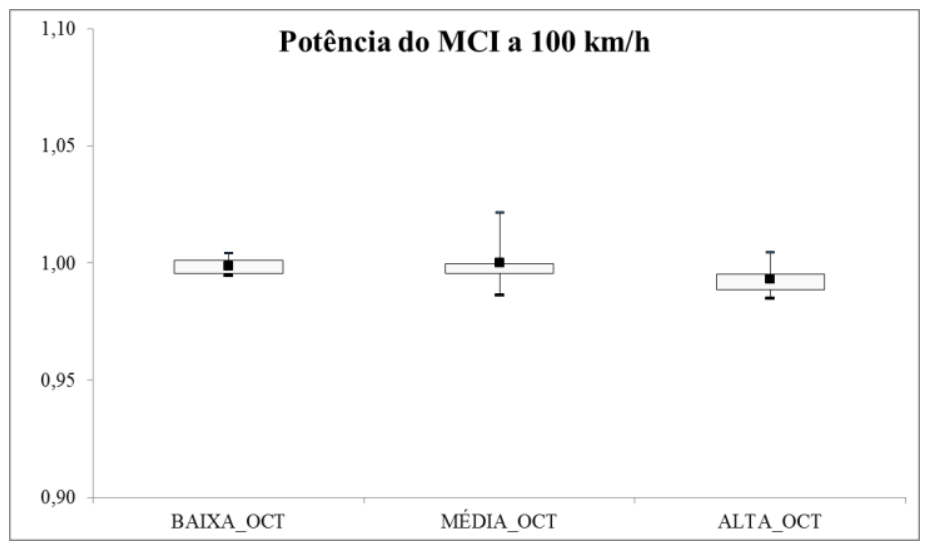

Fig. 12. Resultados dos ensaios de potência do MCI a 100 km/h.

Especificamente na velocidade de $60 \mathrm{~km} / \mathrm{h}$ (figura 8), a gasolina "ALTA_OCT" apresentou uma relativa perda de potência, o que não era esperado para este combustível. Entretanto, como este comportamento não foi verificado nas outras condições de velocidade, assume-se que, de forma global, não houve variações importantes na potência do MCI em função das propriedades do combustível.

\subsection{Consumo Específico em Regime Permanente}

As figuras 13 a 16 apresentam graficamente os resultados para o consumo específico encontrados com os três combustíveis, nas quatro condições de velocidade constante.

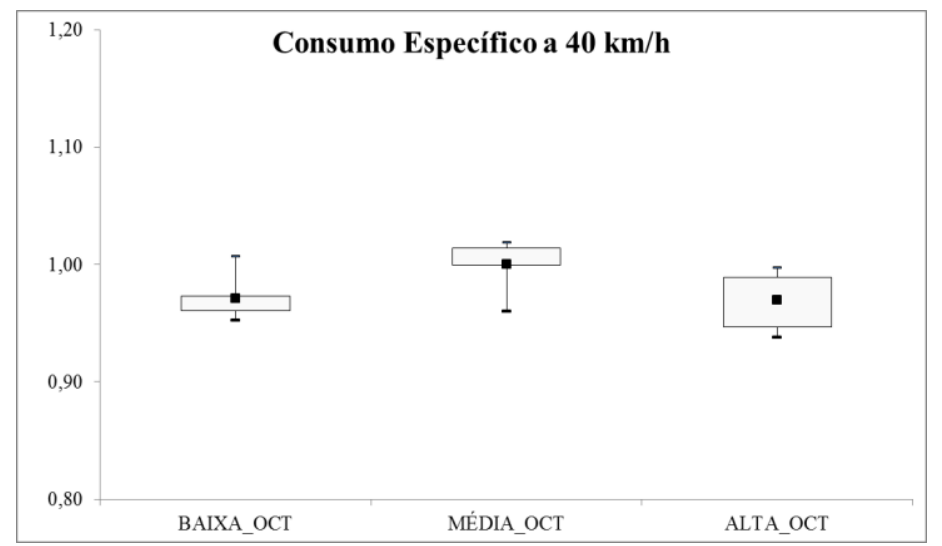

Fig. 13. Resultados dos ensaios de consumo específico a $40 \mathrm{~km} / \mathrm{h}$.

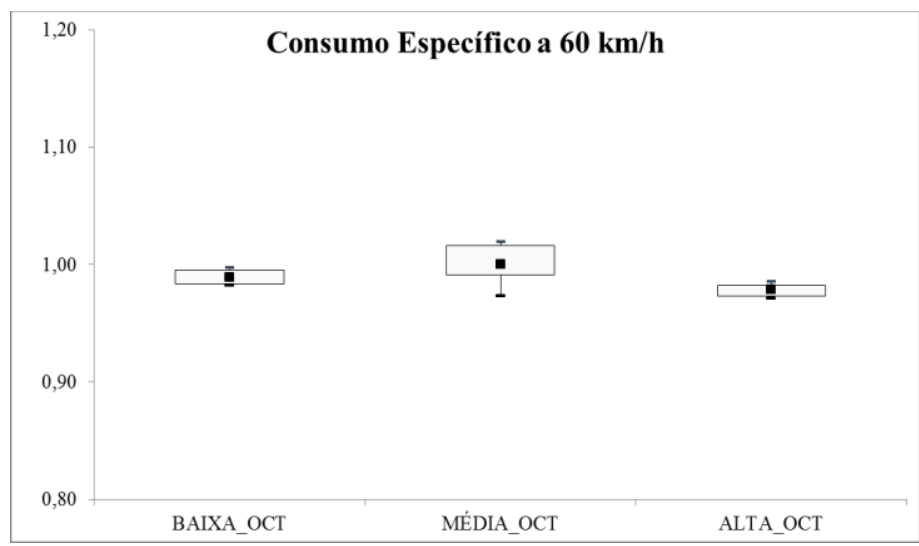

Fig. 14. Resultados dos ensaios de consumo específico a $60 \mathrm{~km} / \mathrm{h}$. 


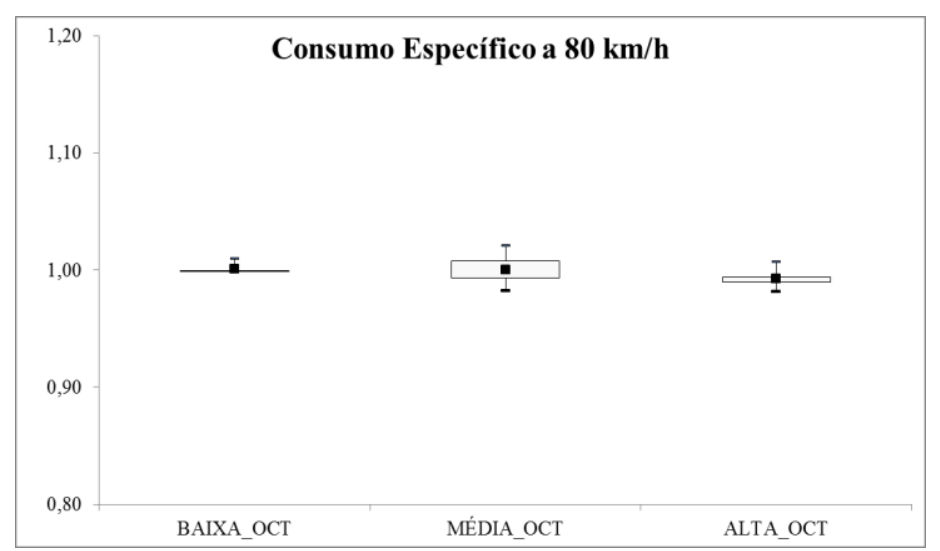

Fig. 15. Resultados dos ensaios de consumo específico a $80 \mathrm{~km} / \mathrm{h}$.

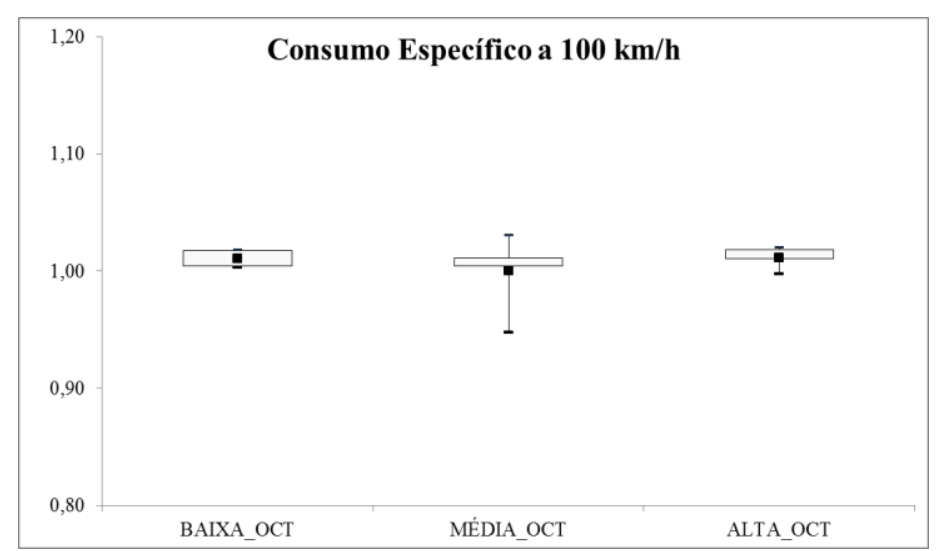

Fig. 16. Resultados dos ensaios de consumo específico a $100 \mathrm{~km} / \mathrm{h}$.

Nota-se destes resultados que não foram encontradas diferenças estatisticamente significativas na comparação das médias, tanto da gasolina "BAIXA_OCT", quanto da "ALTA_OCT" em comparação à gasolina de referência "MÉDIA_OCT".

\subsection{Detonação}

A Tabela 3 agrupa todos os registros obtidos a partir do sensor de detonação do veículo com os três combustíveis, nas quatro condições de velocidade, bem como os registros do avanço de ignição em cada condição.

Pode-se perceber que em nenhum dos casos houve eventos de detonação. É importante ainda salientar que os valores de avanço da ignição praticamente se mantiveram constantes em todas as condições, o que indica que não houve correções da central eletrônica do motor para reduzir o avanço e evitar as detonações.

Este resultado pode indicar que, em função da menor taxa de compressão, resultante do fechamento tardio da válvula de admissão nos motores do ciclo Atkinson, o uso de gasolinas de baixa octanagem nestes motores é mais bem tolerado do que nos motores do ciclo Otto, não trazendo, em princípio, riscos de danos por detonação. 
Tabela 3. Perfis dos sinais do sensor de detonação nos ensaios em regime permanente.

\begin{tabular}{|c|c|c|}
\hline GASOLINA & $\operatorname{AVANÇO~}\left(^{\circ}\right)$ & $40 \mathrm{~km} / \mathrm{h}$ \\
\hline \multirow{2}{*}{ BAIXA_OCT } & \multirow{2}{*}{2} & 100 \\
\hline & & 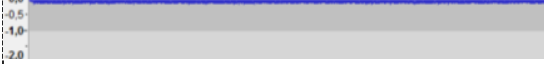 \\
\hline \multirow{3}{*}{ MÉDIA_OCT } & & $\mid$ \\
\hline & \multirow[t]{2}{*}{3} & $\begin{array}{ll}0.5 \\
0.5 \\
0.0 \\
0.5\end{array}$ \\
\hline & & $\begin{array}{l}1,0.0 \\
2.0\end{array}$ \\
\hline \multirow{3}{*}{ ALTA_OCT } & \multirow{3}{*}{2} & 2 \\
\hline & & 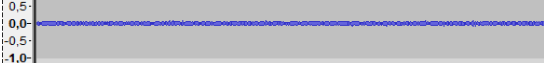 \\
\hline & & $\begin{array}{l}1-1,0.0 \\
12,0\end{array}$ \\
\hline GASOLINA & AVANÇO $\left(^{\circ}\right)$ & $60 \mathrm{~km} / \mathrm{h}$ \\
\hline \multirow{2}{*}{ BAIXA_OCT } & \multirow{2}{*}{3} & $\begin{array}{l}20 \\
105 \\
0.0 \\
0.0 \\
\end{array}$ \\
\hline & & $\begin{array}{l}105 \\
100 \\
20\end{array}$ \\
\hline \multirow[b]{2}{*}{ MÉDIA_OCT } & \multirow[b]{2}{*}{3} & $\mid$ \\
\hline & & 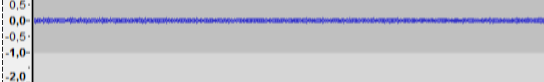 \\
\hline \multirow{3}{*}{ ALTA_OCT } & \multirow{3}{*}{3} & $2 0 \longdiv { 2 }$ \\
\hline & & 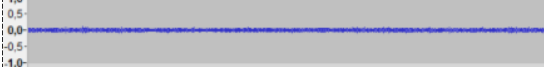 \\
\hline & & 1,120 \\
\hline GASOLINA & AVANÇO $\left(^{\circ}\right)$ & $80 \mathrm{~km} / \mathrm{h}$ \\
\hline \multirow{2}{*}{ BAIXA_OCT } & \multirow{2}{*}{3} & {$\left[\begin{array}{l}100 \\
0.05 \\
0.06\end{array}\right.$} \\
\hline & & 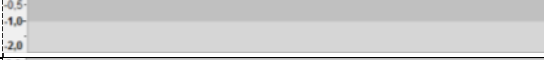 \\
\hline \multirow{2}{*}{ MÉDIA_OCT } & \multirow{2}{*}{3} & 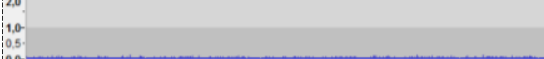 \\
\hline & & 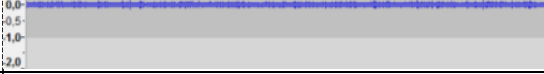 \\
\hline \multirow{2}{*}{ ALTA_OCT } & \multirow{2}{*}{3} & $\begin{array}{l}20.0 \\
10.0 \\
0.00\end{array}$ \\
\hline & & $\begin{array}{l}0.5 \\
120 \\
20\end{array}$ \\
\hline GASOLINA & AVANÇO $\left(^{\circ}\right)$ & $100 \mathrm{~km} / \mathrm{h}$ \\
\hline \multirow{2}{*}{ BAIXA_OCT } & \multirow{2}{*}{3} & 1,08 \\
\hline & & 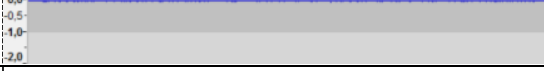 \\
\hline \multirow[b]{2}{*}{ MÉDIA_OCT } & \multirow[b]{2}{*}{3} & 200 \\
\hline & & 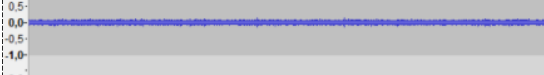 \\
\hline \multirow{3}{*}{ ALTA_OCT } & \multirow{3}{*}{3} & 200 \\
\hline & & 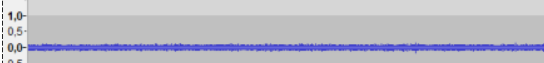 \\
\hline & & 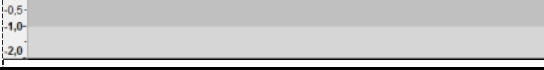 \\
\hline
\end{tabular}

\subsection{Emissões de $\mathrm{CO}_{2}$ e Autonomia Urbana}

A figura 17 apresenta os resultados médios das emissões de $\mathrm{CO}_{2}$. Percebe-se que houve redução com o uso da gasolina de baixa octanagem em relação à gasolina comercial, o que está diretamente relacionado ao menor teor de carbono deste combustível, como 
pode ser verificado na Tabela 2. Entretanto, esta redução nas emissões de $\mathrm{CO}_{2}$ não foi diretamente traduzida em aumento relativo da autonomia urbana.

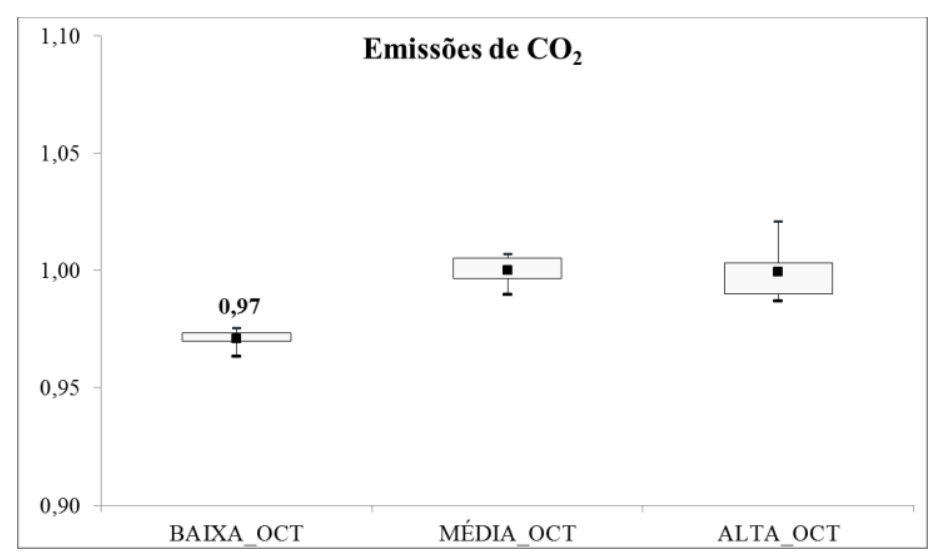

Fig. 17. Resultados dos ensaios de emissões de $\mathrm{CO}_{2}$.

Nota-se na figura 18 que a gasolina BAIXA_OCT gerou perdas de autonomia em relação às demais, o que pode ser explicado pelo valor significativamente menor da sua massa específica em comparação às outras formulações, o que foi preponderante no resultado final do cálculo da autonomia segundo a Equação 1.

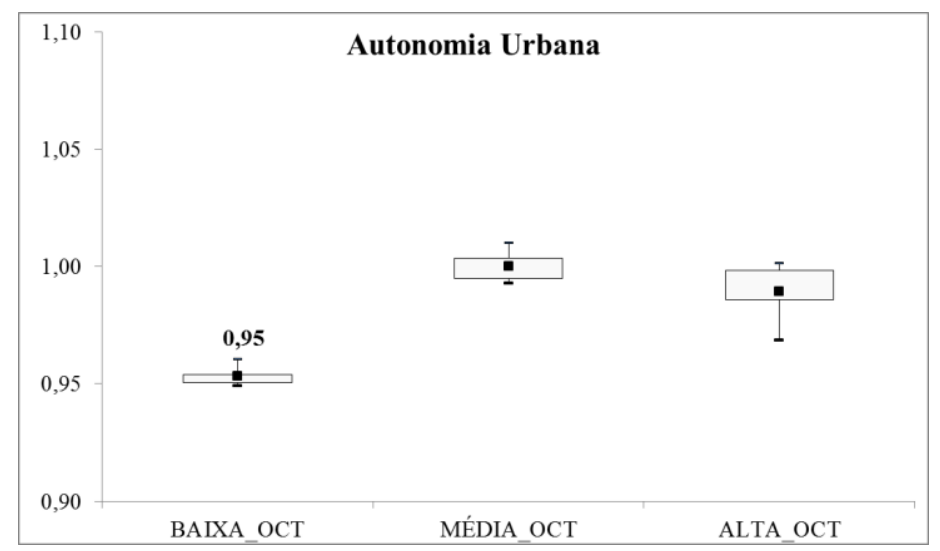

Fig. 18. Resultados dos ensaios de emissões de autonomia urbana.

A partir da observação da Tabela 4, que apresenta um panorama geral de todos os resultados obtidos neste estudo, pode-se concluir que a gasolina de baixa octanagem e baixa densidade "BAIXA_OCT" não apresentou perdas de desempenho em retomadas de velocidade e potência do motor a combustão, bem como não trouxe riscos de ocorrências de detonação, diferentemente do observado em trabalhos anteriores do Cenpes com a mesma gasolina em veículos com motores do ciclo Otto.

Observou-se porém uma tendência de redução nas emissões de $\mathrm{CO}_{2}$ da ordem de $3 \%$ em relação à gasolina de referência "MÉDIA_OCT", que está de acordo com o menor conteúdo de carbono na composição deste combustível, conforme pode ser observado na Tabela $2(73,6 \% \times 75,4 \%)$. Apesar desta redução nas emissões de $\mathrm{CO}_{2}$, obteve-se uma redução também na autonomia urbana da ordem de $5 \%$, que pode ser explicada pela densidade mais baixa deste combustível em relação à gasolina de referência $\left(713,4 \mathrm{~kg} / \mathrm{m}^{3} \times 748,8 \mathrm{~kg} / \mathrm{m}^{3}\right)$. Por outro lado, o uso da gasolina de alta octanagem "ALTA_OCT" não promoveu ganhos significativos em relação ao 
desempenho nem à eficiência do motor quando comparada à gasolina de referência "MÉDIA_OCT".

Tabela 4. Resumo dos resultados obtidos com as gasolinas "BAIXA_OCT" e "ALTA_OCT", em relação à gasolina de referência "MÉDIA OCT".

\begin{tabular}{|l|l|l|}
\hline \multicolumn{1}{|c|}{$\begin{array}{c}\text { Em relação à } \\
\text { Gasolina MÉDIA_OCT }\end{array}$} & BAIXA_OCT & ALTA_OCT \\
\hline Retomadas de Velocidade & Sem diferenças & Sem diferenças \\
\hline Potência do MCI & Sem diferenças & Sem diferenças \\
\hline Consumo Específico & Sem diferenças & Sem diferenças \\
\hline Detonação & Sem diferenças & Sem diferenças \\
\hline Emissões de $\mathrm{CO}_{2}$ & Redução de 3\% & Sem diferenças \\
\hline Autonomia Urbana & Redução de 5\% & Sem diferenças \\
\hline
\end{tabular}

\section{CONCLUSÃO}

Neste trabalho foram investigados os possíveis efeitos de diferentes formulações de gasolinas em um veículo híbrido com o motor do ciclo Atkinson, cuja característica principal é possuir um curso de deslocamento do pistão no tempo de compressão menor do que no tempo da expansão.

Esta característica permite que o calor remanescente da combustão seja mais bem aproveitado em forma de trabalho na expansão, aumentando assim a eficiência térmica de conversão do motor. A diferença entre os cursos de compressão e de expansão é dada pelo fechamento tardio da válvula de admissão, o que resulta em uma taxa de compressão efetiva inferior a que seria obtida com o mesmo motor operando segundo o ciclo Otto.

Três diferentes gasolinas foram testadas, sendo uma com baixa octanagem e baixa densidade, uma gasolina premium, com elevada octanagem e uma terceira com características de octanagem e densidade representativas de uma gasolina comercial, que foi selecionada como referência para comparação.

De forma geral, a partir desta observação, verifica-se que o uso de uma gasolina de baixa octanagem no motor do ciclo Atkinson avaliado, não trouxe riscos significativos de integridade e de funcionamento, nem perdas importantes de desempenho. Foram observadas reduções nas emissões de $\mathrm{CO}_{2}$ e na autonomia urbana, compatíveis respectivamente com o menor conteúdo de carbono e com a densidade mais baixa desta gasolina em relação à gasolina comercial.

Quanto à comparação entre a gasolina de alta octanagem e a gasolina comercial, observou-se que não houve ganhos significativos de desempenho e de eficiência.

Esta foi uma primeira observação neste sentido e é recomendável que este estudo tenha prosseguimento com a avaliação mais detalhada deste e de outros parâmetros do motor, bem como seja estendido para os motores que operam de forma parcial nos ciclos Atkinson e Otto. 


\section{REFERÊNCIAS}

[1] KALGHATGI, G., Is it really the end of internal combustion engines and petroleum in transport?, Applied Energy, Volume 225, pp. 965-974, 2018.

[2] KALGHATGI, G., Development of Fuel/Engine Systems - The Way Forward to Sustainable Transport, Engineering (article in press), Disponível em www.elsevier.com/locate/eng. Acesso em: 20 de maio de 2019.

[3] JOHNSON, T., JOSHI, A., Review of Vehicle Engine Efficiency and Emissions, SAE Technical Paper 2017-01-0907, 2017.

[4] JOHNSON, T., JOSHI, A., Review of Vehicle Engine Efficiency and Emissions, SAE Technical Paper 2018-01-0329, 2018.

[5] MAZDA, SKYACTIV-X: a revolutionary new combustion engine. Disponível em https://www.mazda.com/en/innovation/mazda-stories/engineers/skyactiv-x/. Acesso em: 15 de fevereiro de 2019.

[6] ACHATES POWER, Opposed-Piston - Achates Power. Disponível em http://achatespower.com/our-formula/opposed-piston/. Acesso em: 15 de fevereiro de 2019.

[7] INFINITI. INFINITI VC-Turbo engine: Power \& efficiency optimized. Disponível em https://www.infiniti.co.uk/about-infiniti/campaign-news-events/VC-Turbo.html. Acesso em: 15 de fevereiro de 2019.

[8] HEYWOOD, J. B., 1988, Internal Combustion Engine Fundamentals, New York, McGrawHill.

[9] ATKINSON, J., "Gas Engine”, patente US 367496, 02/08/1887.

[10] VIECELI, D. J., "Nivelando a Engenharia - Motores de combustão interna: parte 1", Disponível em https://nivelandoaengenharia.com.br/geral/otto/. Acesso em: 22/05/2019.

[11] WIKIPEDIA, "Ciclo Atkinson”, Disponível em

https://pt.wikipedia.org/wiki/Ciclo_Atkinson. Acesso em: 20/05/2019.

[12] VILLELA, A. C. S., BOTERO, S. W., CARVALHO, R. N., Vehicle Speed Recovery Test Methodologies on Chassis Dynamometer and their Correlation with Track Test Results, SAE Technical Paper 2015-36-0196, 2015.

[13] ABNT, Veículos rodoviários - Código de ensaio de motores - Potência líquida efetiva, ABNT NBR 1585:1996.

[14] MACHADO, G. B., Metodologias para Desenvolvimento de Combustíveis e Determinação da Velocidade de Propagação de Chama em Motores de Ignição por Centelha, Tese de D.Sc., PUC-Rio, Rio de Janeiro, RJ, Brasil, 2012.

[15] KALGHATGI, G.T., Fuel Anti-Knock Quality - Part I. Engine Studies, SAE Technical Paper 2001-01-3584, San Antonio, Texas, 2001.

[16] ABNT, Veículos rodoviários híbridos elétricos leves - Medição de emissão de escapamento e consumo de combustível e energia - Métodos de ensaio", ABNT NBR 16567:2016. 